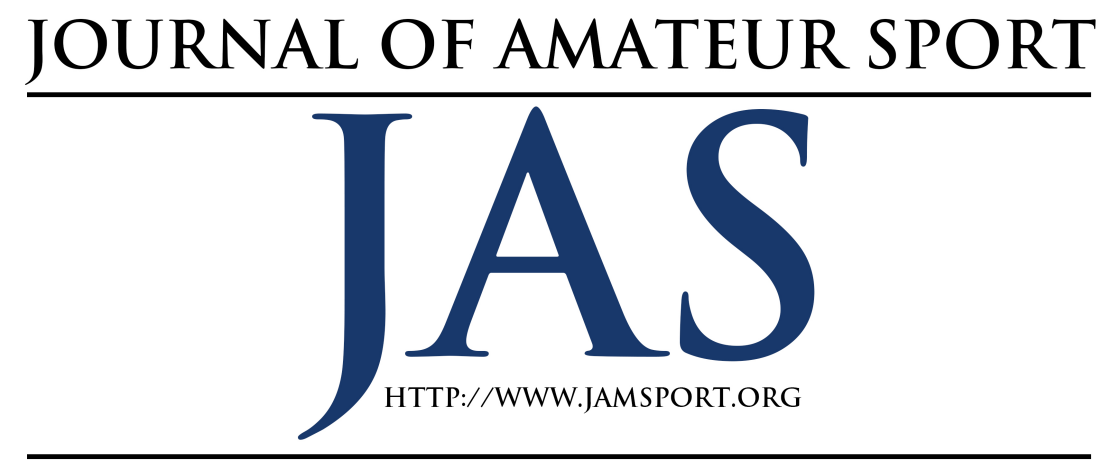

\title{
Mission and Purpose
}

The overarching mission of the Journal of Amateur Sport (JAS) is to provide scholars an outlet in which to share scholarship relevant to the amateur sports realm. We define amateur sport as those who participate and govern at the youth, recreational, community, international, and intercollegiate level. We acknowledge the tenuous debate surrounding the amateurism of intercollegiate athletics, thus at this time we welcome examinations that are focused on the less commercialized avenues of college sport participation and governance (especially NCAA Division II, III, and other less publicized governing bodies and settings). Submissions from all disciplines are encouraged, including sociology, communication, and organizational behavior. Similarly, we welcome a wide array of methodological and structural approaches, including conceptual frameworks, narratives, surveys, interviews, and ethnographies.

As an open-access journal, submissions should be of interest to researchers and practitioners alike. In all, the content published in JAS should advance the collective understanding of the participants, coaches, administrators, and/or institutional structures that comprise amateur sports worldwide. We challenge authors to submit creative and nontraditional manuscripts that are still high-quality in nature. Authors are encouraged to email the editors before submitting if they are unsure if their manuscript is a proper fit within JAS.

\section{Call for Papers}

Thank you for considering the Journal of Amateur Sport (JAS) for your scholarly work. Please follow the guidelines laid out below when submitting your manuscript to JAS. Visit http:/ /www.jamsport.org and click "Submit Now" to begin the submission process. To aid in the double-blind review process, please include three separate files: (1) a title page with corresponding author information, (2) an abstract of no more than 500 words with no identifying information, and (3) the full manuscript with no identifying information. The manuscript should not have been simultaneously submitted for publication or been published previously. Manuscripts should follow 
the current Publication Manual of the American Psychological Association with exception to the elements noted below. The document must be double-spaced, in Garamond font, size 14, and utilize one inch margins throughout. Maximum length, including references and figures, is 50 pages. Be sure to include a running header, page numbers, and footnotes (when appropriate). Authors are responsible for receiving permission to reproduce copyrighted material before submitting their manuscript for publication.

There is no charge for submission or publication. Authors will be provided with a free digital and print copy of published articles. JAS is an open-access, online journal and thus strongly encourages the posting and sharing of published articles by authors on their personal and departmental websites, Google Scholar, and e-portfolios once they are posted to the JAS website. Authors should expect a maximum 60-day turnaround time from initial submission to receiving the initial review. Submissions that are determined to be outside of the scope or not appropriate for JAS are subject to desk rejection. If an article is deemed fit for publication, the author(s) must sign a publishing agreement before the article is officially accepted. Submissions will be subjected to a doubleblind review from at least two members of the editorial board (or outside reviewers when appropriate). 